\title{
Motion Error Analysis and Modeling Technology of CNC Camshaft Grinder
}

\author{
Jinwei Fan, Haohao Tao, Changjun Wu, Yuhang Tang \\ College of Mechanical Engineering \& Applied Electronics Technology, Beijing University of Technology, Beijing100124, China
}

\begin{abstract}
Taking certain CNC camshaft grinder as the subject, its motion error analysis and modeling were studied. The movement forms and types of errors between the moving parts were analysed, the movement between coordinate systems of the adjacent bodies was used to express the movement between the two adjacent bodies, and the ideal motion equation and actual motion equation in case of errors between the adjacent bodies were set up. Actual motion equation between adjacent bodies was made and further extended to analyse arbitrary low-order body arrays, which provides a theoretical basis for studying the multi-branch $\mathrm{CNC}$ camshaft grinding machine error modeling. Complex multi-branch chain CNC camshaft grinder simplified was made as a simple multibody system, and corresponding body coordinate systems and motion reference coordinate systems for moving parts were established to calculate the corresponding transformation matrix between adjacent bodies. Moving parts of the machine were divided into "workpieces-bed" and "wheel-bed" two kinematic chains. The precision constraint equation of the machine is $P_{t}=P_{w}$ in case of using errors influence, and the constraint equation was solved. It provides necessary conditions for study of $\mathrm{CNC}$ camshaft grinder error compensation. Results show that the machine precision is significantly improved after error compensation.
\end{abstract}

\section{Introduction}

High machining precision is critically important and has increased demand in recent years in aerospace, military, ships, cars, and other industries [1,9]. CNC camshaft grinder as an efficiency, high-precision machining equipment of the camshaft which precision retaining ability is essential. However, Due to the existence of different kinds of error source, all machine tools will be faced with the problem of lower accuracy. It is necessary to analyze the errors of machine tools and take appropriate measures to realize error compensation. Therefore, the method of reducing the effect of error on the machining precision of machine tool can be divided into error prevention and error compensation. By establishing the error model of machine tools, identifying all errors of machine tools and reducing the influence of machine errors on machining accuracy by means of correcting NC instructions, which is called error compensation to reduce the effect of the error of machine tool on the machining precision of the workpiece. The method can overcome the shortcomings of the traditional error prevention method, such as high cost and poor applicability, so it has been developed rapidly.

The kinematic error analysis and modeling of machine tools are the basis of error compensation, the analysis of kinematic error is the key of error modeling. In order to improve the machining accuracy and precision retaining ability of the $\mathrm{CNC}$ camshaft grinder, the kinematic error analysis and modeling of the $\mathrm{CNC}$ camshaft grinder was proposed. At present, considerable research work for error compensation of multi-axis machine tools is devoted. Su[1] adopted the multi body system (MBS) theory for the precision modeling and error compensation of multi axis CNC machine tools. Fan et al.[2] studied the geometric error compensation technique for improving the accurcy of precision cam grinding. According to ref.[3], the software design of the error analysis and compensation technology for the camshaft NC grinding are carried out. Wang[4]adopted polynomial fitting and linear fitting method to the geometric error and thermal error modeling of $\mathrm{CNC}$ machine tools and the on-line error compensation. According to ref.[5], the geometric error compensation and error traceability of $\mathrm{CNC}$ machine tools were analyzed. Lechniak et al.[6] proposed the offline software error compensation method. Chnan et al.[7] presented a error compensation method for geometric error and force error of three-axis grinding machine. Zhang et al.[8] used the double ball bar to detect and compensate the error of the rotary table of five axis CNC machine tool. Machine tools are regarded as complex mechanical systems which made up of multiple moving bodies[10]. In recent years, along with the application of follow-up type camshaft grinder is more extensive, in addition, the error analysis and modeling method is too complex and lack of general model. Therefore, the theory of multi-body system can be used to reduce the difficulty of the error modeling of machine tool. However, the multi body system theory is seldom applied to the error modeling of machine tool. So this 
paper uses the theory of multi-body system to establish model of follow-up type camshaft grinder geometry error.

\section{Kinematic error analysis of CNC camshaft grinder}

In this study, a CNC camshaft grinder is chosen as the research object, as shown in Fig. 1. The machine tool consists of machine bed (body 1), Z-axis slide carriage (body 2), grinding wheel heads (body 3 ), spindle (body 4), workpiece (body 5), X-axis slide carriage (body 6), grinding wheel frame (body 7), grinding wheel (body 8) and tailstock (body 9). The grinding wheel moves along the $\mathrm{X}$-axis, worktable moves along the $\mathrm{Z}$-axis, and the spindle rotation around the $\mathrm{C}$-axis. The cam profile is accomplished by simultaneously controlling the $\mathrm{X}$-axis and the C-axis. Geometric errors are usually composed of position-independent geometric errors (PIGEs), and position-dependent geometric errors (PDGEs). PIGEs don't vary with the moving position of the machine tool, but PDGEs are the opposite ones. Fig. 2 is the schematic diagram of PIGEs between linear axes. Based on the theory of the rigid body motion, each moving part of a machine tool has six DOFs in the Cartesian coordinate system. Hence, each moving axis of the CNC camshaft grinder has six PDGEs. Fig.3 shows six PDGEs of X-axis, where $\delta_{i}(x)$ and $\varepsilon_{i}(x)(i=x, y, z)$ represent the linear errors and the angular errors of $\mathrm{X}$-axis along the $i$ direction respectively. Therefore, all the geometric errors of the CNC camshaft grinder are listed, as shown in the Table 1 .

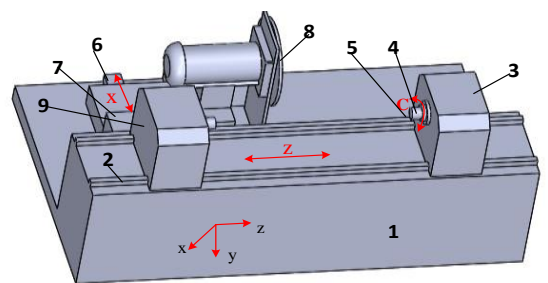

Fig. 1 Structure map of CNC camshaft grinder

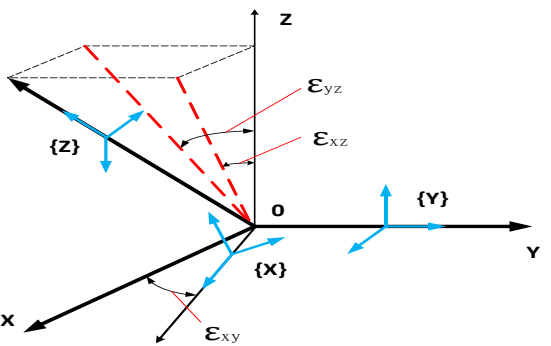

Fig.2 the schematic diagram of PIGEs between linear axes

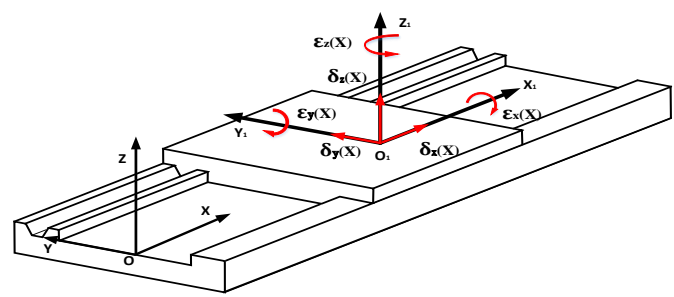

Fig.3 The schematic diagram of PDGEs of X-axis

\section{Topological structure and lower body array}

Based on MBS theory, a machine tool can be assumed to be composed of various rigid bodies. Fig.4 shows the topology structure map of the CNC camshaft grinder, where " $B_{i}$ " means the $i$-th body. As shown in Fig. 4 , the kinematic chain of the $\mathrm{CNC}$ camshaft grinder can be divided into two branches. One is workpiece branch ( $B_{1}$ $B_{2}-B_{3}-B_{4}-B_{5}$ ), and the other is wheel branch ( $B_{1}$ $\left.B_{6}-B_{7}-B_{8}\right)$.

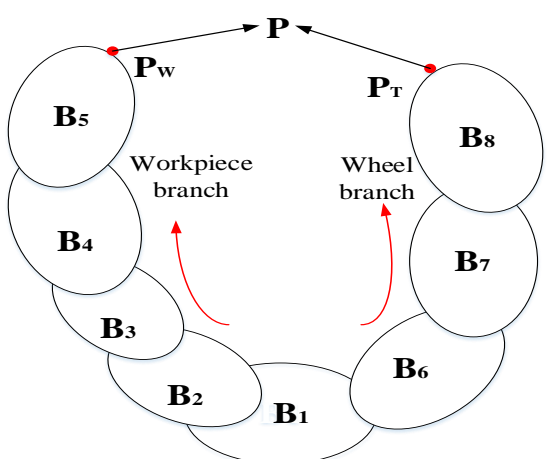

Fig.4 the topology structure of the CNC camshaft grinder

\section{Establishment of the relationship between adjacent body motion}

Suppose that the workpiece and tool are clamped without errors. Point $\mathrm{O}$ is origin of $B_{1}$ body coordinate and also base origin of the machine. When $j=1,2,3,4,5,6, Q_{j}^{1}$ and $Q_{j}$ are ideal and actual motion reference points of $B_{j}$, respectively. $O_{j}^{1}$ and $O_{j}$ are ideal and actual position origin of $B_{j}$ body coordinate in $Q_{j}$ coordinate system. Thus, $q_{j}^{1}$ and $q_{j}$ are ideal and actual position vectors of $B_{j}$ relative to $B_{j-1}$ and $q_{j}^{e}$ is position error vector. $S_{j}^{1}$ and $S_{j}$ are ideal and actual motion vectors of $B_{j}$ relative to $B_{j-1}$ and $S_{j}^{e}$ is displacement error. $q_{w}$ is position matrix of workpiece relative to the worktable and $q_{t}$ is position matrix of tool relative to B-axis. $r_{w}$ and $r_{t}$ are position vectors of tool center in the workpiece coordinate system and tool coordinate system. 
Table. 1 Geometric errors of the CNC camshaft grinder

\begin{tabular}{llllllll}
\hline & & \multicolumn{2}{l}{$\begin{array}{l}\text { Linear } \\
\text { error }\end{array}$} & \multicolumn{5}{c}{$\begin{array}{l}\text { Angular } \\
\text { error }\end{array}$} \\
\cline { 3 - 8 } & Axis & $\begin{array}{l}\mathrm{X} \\
\text { direction }\end{array}$ & $\begin{array}{l}\mathrm{y} \\
\text { direction }\end{array}$ & $\begin{array}{l}\mathrm{Z} \\
\text { direction }\end{array}$ & Around $\mathrm{x}$ & Around & Around \\
& & $\delta_{x}(x)$ & $\delta_{y}(x)$ & $\delta_{z}(x)$ & $\varepsilon_{x}(x)$ & $\varepsilon_{y}(x)$ & $\varepsilon_{z}(x)$ \\
\hline PDGES & $\mathrm{X}$ & $\delta_{y}(z)$ & $\delta_{z}(z)$ & $\varepsilon_{x}(z)$ & $\varepsilon_{y}(z)$ & $\varepsilon_{z}(z)$ \\
& $\mathrm{Z}$ & $\delta_{x}(z)$ & $\delta_{y}(c)$ & $\delta_{z}(z)$ & $\varepsilon_{x}(\mathrm{c})$ & $\varepsilon_{y}(\mathrm{c})$ & $\varepsilon_{z}(\mathrm{c})$ \\
& $\mathrm{C}$ & $\delta_{x}(c)$ & & & & $\varepsilon_{x z}, \varepsilon_{x c}$ & $\varepsilon_{z \mathrm{c}}$ \\
\hline
\end{tabular}

\section{Kinematic error modeling of CNC camshaft grinder}

The CNC camshaft grinder is a special multi body system with only two branches, and the components are

$$
\begin{gathered}
P_{w}=P_{w o}=S_{21} S_{32} S_{43} S_{54} S_{5 r}=S_{12 p} S_{12 p e} S_{12 s} S_{12 s e} S_{23 p} S_{23 p e} S_{23 s} S_{23 s e} S_{34 p} S_{34 p e} S_{34 s} S_{34 s e} S_{45 p} S_{45 p e} S_{45 s} S_{45 s e} S_{5 r} \\
P_{t}=P_{t o}=S_{61} S_{76} S_{87} S_{8 r}=S_{16 p} S_{16 p e} S_{16 s} S_{16 s e} S_{67 p} S_{67 p e} S_{67 s} S_{67 s e} S_{78 p} S_{78 p e} S_{78 s} S_{78 s e} S_{8 r}
\end{gathered}
$$

$$
P_{w}=P_{t}
$$

where $P_{w}$ is actual grinding position in workpiece branch and $P_{t}$ is actual Grinding position in tool branch. $S_{i j p}$ is ideal position matrices, $S_{i j s}$ is ideal motion matrices. $S_{i j p e}$ is actual position matrices, $S_{i j s e}$ is actual motion matrices. $S_{5 r}$ represent position matrix of actual grinding point in workpiece branch, $S_{8 r}$ represent position matrix of actual grinding point in tool branch. An important ideal condition is that the actual grinding position in workpiece branch is equal to the actual grinding position in tool branch, as shown in Eq. 5,

Table 2. The D-H matrices of the CNC Camshaft Grinder (parts)
Suppose that the position matrix of the coordinate system of the workpiece relative to the coordinate system position matrix of the coordinate system of the tool relative to the coordinate system of the spindle faces is $\left(\begin{array}{llll}q_{8 x} & q_{8 y} & q_{8 z} & 1\end{array}\right)^{T}$. Let $\mathrm{E}$ represents a unit matrix. Then, D-H matrices can be built, which are shown in Table 2. of the spindle faces is $\left(\begin{array}{lllll}q_{5 x} & q_{5 y} & q_{5 z} & 1\end{array}\right)^{T}$, the

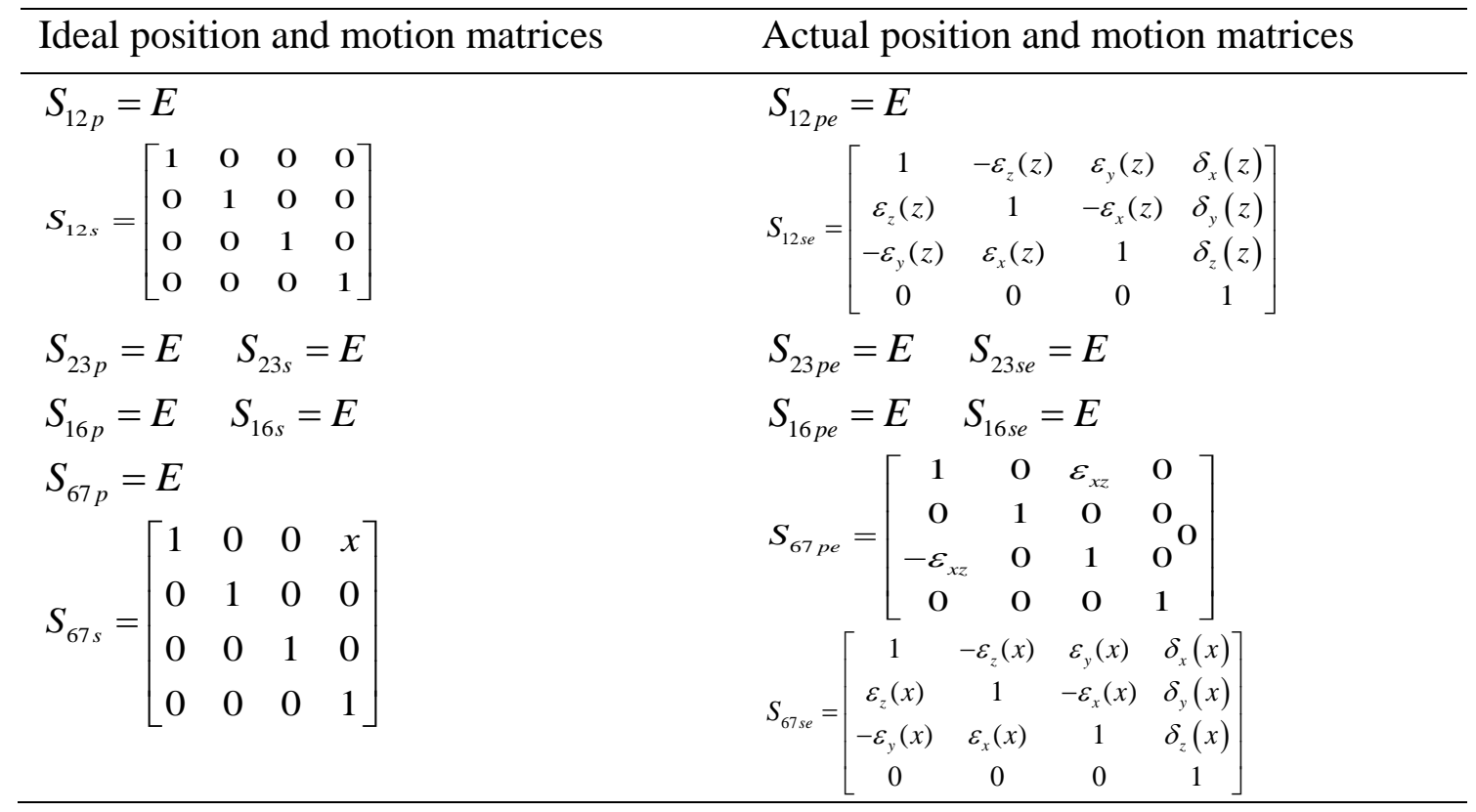


Transform D-H matrices into the Eqs. ((3)-(5)), then the precision machining constraint equation of $\mathrm{CNC}$

$$
\begin{gathered}
\delta_{x}(z)+q_{5 z}\left(\varepsilon_{x c}+\varepsilon_{y}(z)+\varepsilon_{x}(c) \sin c-\varepsilon_{y}(c) \cos c\right)-q_{5 y}\left(\sin c+\varepsilon_{z}(x) \cos c+\cos c\right)-q_{5 x}\left(\varepsilon_{z}(x) \sin c-\cos c-\varepsilon_{z}(c) \sin c\right) \\
-x_{w}\left(\varepsilon_{z}(x) \sin c-\cos c+\varepsilon_{z}(c) \sin c\right)-y_{w}\left(\sin c+\varepsilon_{z}(x) \cos c+\varepsilon_{z}(c) \cos c\right)+z_{w}\left(\varepsilon_{x c}+\varepsilon_{y}(z)-\varepsilon_{x}(c) \sin c+\varepsilon_{y}(c) \cos c\right) \\
+\delta_{x}(c) \cos c-\delta_{y}(c) \sin c=x+q_{8 x}+x_{t}+\delta_{x}(x)+z \varepsilon_{x z}-q_{8 y} \delta_{z}(x)-y_{t} \varepsilon_{z}(x)+q_{8 z}\left(\varepsilon_{x z}+\varepsilon_{y}(x)\right)+z_{t}\left(\varepsilon_{x z}+\varepsilon_{y}(x)\right) \\
\delta_{y}(z)-q_{5 z}\left(\varepsilon_{y c}+\varepsilon_{x}(z)-\varepsilon_{x}(c) \cos c-\varepsilon_{y}(c) \sin c\right)-q_{5 y}\left(\varepsilon_{z}(z) \sin c+\varepsilon_{z}(c) \sin c-\cos c\right)-q_{5 x}\left(\varepsilon_{z}(x) \cos c\right. \\
\left.+\sin c+\varepsilon_{z}(c) \cos c\right)+x_{w}\left(\varepsilon_{z}(z) \cos c+\sin c+\varepsilon_{z}(c) \cos c\right)-y_{w}\left(\varepsilon_{z}(z) \sin c-\cos c+\varepsilon_{z}(c) \sin c\right)-z_{w}\left(\varepsilon_{y c}+\right. \\
\left.\varepsilon_{x}(z)-\varepsilon_{x}(c) \cos c+\varepsilon_{y}(c) \sin c\right)+\delta_{x}(c) \sin c+\delta_{y}(c) \cos c=y_{t}+q_{8 y}+\delta_{y}(x)-\left(q_{8 z}+z_{t}\right) \varepsilon_{x}(x)+\left(q_{8 x}+x_{t}\right) \varepsilon_{z}(x) \\
z+\delta_{z}(z)+\delta_{z}(c)-q_{5 z} \varepsilon_{y}(c) \cos c+q_{5 y}\left(\left(\varepsilon_{x c}+\varepsilon_{y}(z)\right) \sin c+\left(\varepsilon_{y c}+\varepsilon_{x}(x)\right) \cos c+\varepsilon_{x}(c)\right)+ \\
q_{5 x}\left(\left(\varepsilon_{y c}+\varepsilon_{x}(z)\right) \sin c-\left(\left(\varepsilon_{x c}+\varepsilon_{y}(z)\right) \cos c-\varepsilon_{y}(c)\right)+x_{w}\left(\left(\varepsilon_{y c}+\varepsilon_{x}(z)\right) \sin c-\right.\right. \\
\left.\left(\varepsilon_{x c}+\varepsilon_{y}(z)\right) \cos c-\varepsilon_{y}(c)\right) y_{w}\left(\left(\varepsilon_{x c}+\varepsilon_{y}(z)\right) \sin c+\left(\varepsilon_{y c}+\varepsilon_{x}(z)\right) \cos c+\varepsilon_{x}(c)\right)+z_{w}= \\
z+q_{8 z}+z_{t}+\delta_{z}(x)-x \varepsilon_{x z}+\left(q_{8 y}+y_{t}\right) \varepsilon_{x}(x)-q_{8 x}\left(\varepsilon_{x z}+\varepsilon_{y}(x)\right)-x_{t}\left(\varepsilon_{x z}+\varepsilon_{y}(x)\right)
\end{gathered}
$$

In the model of the linkage between the $\mathrm{X}$ axis and the $\mathrm{C}$ axis of machine tools, $z^{\prime} 、 z^{\prime \prime}$ represent the camshaft outline curve, grinding wheel radius is $\mathrm{R}$, the rotation angle of the grinding wheel is $\theta$. According to the ref. [1], the position matrix of actual grinding point in workpiece branch and in tool branch can be obtained, $S_{8 r}=\left[-|R \cos (\arcsin (\rho \sin (\theta-\varphi) / R))|, \rho \sin (\varphi-\theta), z^{\prime \prime}, 1\right]^{T}$ $S_{5 r}=\left[|\rho \cos \varphi|,-\rho \sin \varphi, z^{\prime}, 1\right]^{T}$, Where $\varphi$ represent the cam rotation angle, $z^{\prime} 、 z^{\prime \prime}$ represent the $Z$ axis coordinate values of the grinding point $\mathrm{P}$ in the workpiece coordinate system and in the tool coordinate system, respectively.

The 21 error parameters in the precision machining constraint equation can be measured and identified by the dual ball bar instrument and multi-body system theory. By mapping the relationship between tool route and NC instruction, and between $\mathrm{NC}$ instruction and practical tool path, the NC instructions under the condition of error can be corrected. The machine tool after error compensation was chosen as the research object to process the automobile engine camshaft, the maximum error has been dropped from $22 \mu \mathrm{m}$ to $9 \mu \mathrm{m}$, thus, the machining accuracy of the machine tool after error compensation have been significantly improved.

\section{Conclusion}

Based on the motion condition of the tilting head of five-axis machine tools, three measurement patterns in $\mathrm{Y}$ direction, $\mathrm{X}$ direction and $\mathrm{Z}$ direction are proposed respectively using DBB . Then, the relative displacement equations between two balls of DBB in measurement patterns in $\mathrm{Y}$ direction, $\mathrm{X}$ direction and $\mathrm{Z}$ direction are established respectively on the basis of HTM and MBS theory. Finally, the geometric error parameters of the tilting head (B-axis) are identified totally. The presented method in this paper is universal and provides a reference for the error identification for the tilting head of five-axis machine tools.

\section{Acknowledgments}

This work is financially supported by the National Natural Science Foundation of China (No. 51275014), Science and Technology Major Projects of High-end CNC Machine Tools and Basic Manufacturing Equipment of China (No.2014ZX04011031).

\section{References}

1. LIU Y J, FAN J W, LI Y, et al. Manufacturing precision reliability maintenance technology of the CNC camshaft grinder[J]. Journal of Beijing University of Technology, 2013,39(10):1446-1451.

2. HU Y. The research on geometric error software compensation method of the SK-21 CNC grinding tool[D]. Beijing: Beijing University of Technology, 2006.

3. SU S P. Study on the methods of precision modeling and error compensation for multi-axis CNC machine tools [D]. Changsha: National University of Defense Technology, 2002.

4. FAN J W, GUAN J L, YAN S Z. Technology for enhancing the grinding accuracy of precision cam by geometric error compensation method [J]. China Mechanical Engineering,2004, 15(14) :1223-1226.

5. LI J. Software design and technical study of error analysis and compensation of camshaft NC grinding [D]. Changsha: Hunan University, 2009.

6. WANG W, YANG J G, YAO X D, et al. Synthesis modeling and real-time compensation of geometric error and thermal error for CNC machine tools [J]. Journal of Mechanical Engineering, 2012, 48(7):165-170, 179.

7. WANG X F. Geometric error compensation and error influence tracing analysis for complex machine tool [D]. Beijing: Beijing University of Technology, 2014.

8. LECHNIAK Z, WERNER A, SKALSKI K, et al. Methodology of off-line software compensation for errors in the machining process on the CNC machine tool [J]. Journal of Materials Processing Technology, 1998, 76( $1 / 2 / 3)$ : 42-48. 
9. Wu C, Fan J, Wang Q, Chen D (2018) Machining accuracy improvement of non-orthogonal five-axis machine tools by a new iterative compensation methodology based on the relative motion constraint equation. Int J Mach Tools Manuf. 124(1):80-98
10. Wu CJ, Fan JW, Wang QH, Pan R, Tang YH, Li ZS (2018) Prediction and compensation of geometric error for translational axes in multi-axis machine tools. Int J Adv Manuf Technol. 95:3413-3435. 